Published in final edited form as:

Immunol Rev. 2014 March ; 258(1): 145-149. doi:10.1111/imr.12153.

\title{
Innate allorecognition
}

Martin H. Oberbarnscheidt ${ }^{1,2}$ and Fadi G. Lakkis ${ }^{1,2,3,4}$

${ }^{1}$ Thomas E. Starzl Transplantation Institute, University of Pittsburgh School of Medicine \& University of Pittsburgh Medical Center, Pittsburgh, PA, USA

Department of Surgery, University of Pittsburgh School of Medicine \& University of Pittsburgh Medical Center, Pittsburgh, PA, USA

${ }^{3}$ Department of Immunology, University of Pittsburgh School of Medicine \& University of Pittsburgh Medical Center, Pittsburgh, PA, USA

${ }^{4}$ Department of Medicine, University of Pittsburgh School of Medicine \& University of Pittsburgh Medical Center, Pittsburgh, PA, USA

\section{Summary}

Vertebrates mount strong adaptive immune responses to transplanted organs (allografts), but the mechanisms by which the innate immune system initiates this response are not completely understood. In anti-microbial immunity, non-self molecules associated with pathogens but not present in the host induce the maturation of innate antigen-presenting cells (APCs) by binding to germ-line-encoded receptors. Mature APCs then initiate the adaptive immune response by presenting microbial antigen and providing costimulatory signals to T cells. How allografts activate APCs, however, is less clear, because allografts are presumably sterile. A widely accepted view is that inflammatory or 'danger' molecules released by dying graft cells at the time of transplantation trigger APC maturation and the T-cell response that follows.

Alternatively, it has been proposed that the introduction of microbial products during the surgical procedure could also alert the innate immune system to the presence of the transplanted organ. Here we review why these hypotheses fail to fully explain how the alloimmune response is initiated after transplantation and summarize evidence that recognition of allogeneic non-self by monocytes is a key event in triggering alloimmunity and graft rejection.

\section{Keywords}

allorecognition; innate immune system; transplantation; monocytes; dendritic cells

\section{Introduction}

Vertebrate immunity is dependent on the innate and adaptive immune systems, whose principal function is to protect the host against infection (1). While cells of the innate immune system express germ-line encoded pattern recognition receptors (PRRs) that detect conserved pathogen-associated molecular patterns (PAMPs) on microbes $(2,3)$, cells of the adaptive immune system express a highly diverse repertoire of re-arranging antigen receptors that recognize microbial antigen with great molecular specificity. Immune responses against microbes begin with the activation of innate immune cells by PAMPs.

Correspondence to: Fadi G. Lakkis University of Pittsburgh Thomas E. Starzl Transplantation Institute Biomedical Science Tower W1548 200 Lothrop Street Pittsburgh, PA 15261 Tel.: +1 4123835774 Fax: +1 4123839990 lakkisf@upmc.edu. 
These cells then mature into potent antigen presenting cells (APCs) that activate T lymphocytes, leading to protective immunity against infection.

Vertebrate immune systems are also capable of discriminating between self and non-self tissues of the same species, a phenomenon known as allorecognition (4). Allorecognition has been studied in the context of the rejection of transplanted cells, tissues, and organs as well as graft-versus-host disease after bone marrow transplantation (5). It also occurs under natural conditions, such as the maternal response to paternal antigens on fetal cells and the rejection of transmissible allogeneic tumors (6-8). T cells are the principal cell type of the adaptive immune system responsible for allorecognition, detecting molecular variations in MHC molecules and/or the endogenous peptides bound to them (9). It is less clear how the innate immune system senses allogeneic non-self to cause APC maturation and initiate Tcell activation. In this review, we briefly summarize knowledge in this area and review recent evidence for recognition of allogeneic non-self by innate immune cells and how this form of recognition triggers the anti-graft $\mathrm{T}$-cell response.

\section{Innate immune recognition of microbial non-self}

In 1989 Charles Janeway postulated that non-self recognition extends beyond somatically rearranged receptors on $\mathrm{T}$ and $\mathrm{B}$ lymphocytes to germ line-encoded PRRs present on innate immune cells (10). These PRRs enable innate immune cells to recognize PAMPs, which are not expressed by host cells and therefore are non-self, and to trigger an innate defense against pathogens as well as maturation of APCs. The latter activate the adaptive immune system by presenting antigen and providing costimulation to $\mathrm{T}$ cells. This hypothesis was soon followed by the discovery of the lipopolysaccharide (LPS) receptor, Toll-like receptor 4 (TLR4)(11, 12). Today, several families of PRRs are known, the most extensively studied being the TLR family responsible for detecting a whole host of microbial products (13). Here we address the question of whether the concept of microbial non-self recognition by the innate immune system extends to the detection of allogeneic non-self.

\section{Allogeneic non-self}

In transplantation, an alloantigen is any genetically encoded polymorphic protein that differs between the donor and recipient. Alloantigens are generally divided into two groups: major histocompatibility complex (MHC) and minor histocompatibility antigens (5). MHC proteins are highly polymorphic, ubiquitously expressed, and can elicit a large polyclonal Tcell response. Minor antigen are unrelated to the MHC and include virtually any polymorphic protein that differs between individuals of the same species (14). A single minor antigen elicits a more limited T-cell response than a single MHC mismatch, but the numerous minor antigens present in transplantation are sufficient to trigger rapid graft rejection, even if MHC loci are matched. Allogeneic non-self is therefore comprised of both polymorphic MHC and non-MHC proteins that could potentially be recognized by the innate immune system as well. It is also possible that host innate immune cells react to non-self tissues by sensing non-polymorphic molecules whose expression level or post-translational modification (for example, through glycosylation) differs between donor and recipient.

\section{How is the allograft sensed by the host's innate immune system? Danger hypothesis}

Innate immune cells are activated within minutes to hours after allogeneic transplantation and are thought to respond to inflammatory ligands released by stressed or dying cells (15). These inflammatory ligands are common to self and non-self tissues and have been referred to as danger or damage associated molecular patterns (DAMPs) $(16,17)$. DAMPs are 
diverse cell products that cause inflammation by signaling through TLRs or inflammasomes in innate cells (18-21). A common DAMP is high-mobility group box protein B1 (HMGB1), a nuclear DNA binding protein that is important for chromatin organization and regulation of transcription (22). It is also an inflammatory mediator secreted by dendritic cells (DCs), monocytes, and macrophages and binds to TLR4 (18). Blocking endogenous HMGB1 delayed rejection, reduced intragraft inflammation, and impaired T-helper 1 (Th1) cell immunity in a mouse heart transplantation model (23). Another example of a DAMP is uric acid, which, when present in supra-physiological amounts in the extracellular space, acts as an immune adjuvant that stimulates DC maturation and promotes T-cell activation $(24,25)$. These findings suggest that endogenous danger molecules that are released at the time of transplantation promote inflammation and immunity, but as discussed later, it is unclear whether they are necessary or sufficient for inducing full DC activation required for initiating the adaptive immune responses.

\section{Microbial hypothesis}

It has also been proposed that ubiquitous microbial products (for example, LPS) introduced during the transplantation procedure or released from gut commensals secondary to anesthesia and surgery contribute to DC maturation and graft rejection (26). This hypothesis was initially supported by experiments showing that deletion of the adapter molecule myeloid differentiation factor 88 (Myd88), which is required for signaling by most TLRs, prevents the rejection of single minor antigen-mismatched grafts (27), but as highlighted below, this finding did not hold up in more stringent transplantation models. Nevertheless, several studies have provided evidence that bacterial infection or the administration of TLR ligands enhances alloimmune responses and breaks long-term allograft survival in mouse models of transplantation tolerance. For example, TLR agonists abrogated prolongation of skin allograft survival while the absence of Myd88 signaling promoted allograft acceptance in mice treated with agents that block T-cell costimulation $(28,29)$. Likewise, infecting mice with Listeria monocytogenes broke established tolerance in a mouse heart transplantation model (30). It has also been shown that TLR7 agonists administered to the skin cause local graft-versus-host disease in a bone marrow transplantation model (31). These studies therefore provide evidence that microbial products or infection itself aggravate alloimmune responses but do not prove that PAMPs are responsible for initiating APC maturation and Tcell activation after transplantation.

\section{Shortcomings}

Although inflammation (danger) and microbial ligands present at the time of transplantation enhance the adaptive alloimmune response, it does not appear that either is essential for initiating the response. Immune reactions against grafts that differ from the host by major or multiple minor histocompatibility antigens proceed unabated, even when key innate signaling pathways or cytokines necessary for danger and microbial recognition are inhibited (32-36). In addition, allografts parked in T-cell-deficient hosts until tissue injury and microbial contamination that occurred at the time of transplantation have resolved, are uniformly rejected when the host is replenished with T cells (37-41). These observations imply that innate stimuli may not be absolutely required for rejection, that unaccounted for microbe-derived innate stimuli are present in lymphopenic mice (42), or that additional innate stimulate, other than danger and microbial molecules, may exist in the setting of organ transplantation. Below we provide evidence in support of the latter hypothesis.

\section{Does the innate immune system sense allogeneic non-self?}

Over the past several years, we have tested the possibility that the mouse innate immune system may actually distinguish between self and allogeneic non-self as it does between self 
and microbial non-self, and whether this could be an important trigger of APC maturation and T-cell activation after transplantation. Here we summarize published and unpublished data that support this hypothesis.

\section{Sensing of allogeneic non-self by monocytes}

Compelling evidence that cells of the innate immune system distinguish between self and allogeneic non-self was first provided by Zecher et al (43). Transplantation studies in $R A G^{-/-}$mice, which lack $\mathrm{T}$ and $\mathrm{B}$ lymphocytes, demonstrated that injecting allogeneic splenocytes into the ear pinnae elicited significantly greater swelling and infiltration of the skin with host myeloid cells than injecting syngeneic splenocytes. Prior priming with alloantigen was required for mounting a maximal response to allogeneic cells, and the response exhibited a memory-like feature as it could be elicited four weeks after immunization. Moreover, the response had crude specificity, in that donor-specific recall responses were greater than third party responses. A series of depletion and cell transfer experiments established that the response is independent of NK cells but, instead, was mediated by monocytes. The monocyte response to allogeneic non-self in this model was a dependent on non-MHC rather than MHC disparities between the donor and recipient. Therefore, this study provided strong hints that the mouse innate immune system is capable of allorecognition and that this phenomenon is mediated by monocytes. An additional study published by Li and colleagues (44) also suggested that distinct mechanisms exist by which cells of the monocyte lineage, macrophages to be specific, identify and kill non-self cells. They found that in naive mice NK cells are the primary effector cells responsible for the killing of allogeneic cells via 'missing self' recognition. However, in alloantigenpresensitized mice, NK cells became dispensable. Instead, macrophages appeared to be the principal mediator of allogeneic cell rejection, but this function required help from activated $\mathrm{CD} 4^{+} \mathrm{T}$ cells.

Sensing allogeneic non-self by monocytes triggers DC maturation and graft rejection Although the above studies indicated that the mouse innate immune system is capable of distinguishing between self and non-self cells, they did not determine whether such innate sensing leads to the generation of mature DC that then activate T cells and cause graft rejection. In unpublished work, we have demonstrated that vascularized heart grafts transplanted to $\mathrm{RAG}^{--} \gamma \mathrm{c}^{-/-}$mice, which lack T, B, and NK cells, are rapidly infiltrated by host monocyte-derived DCs (mono-DCs) that take on a mature phenotype (high expression of costimulatory and MHC class II molecules). However, distinct differences between mono-DCs that infiltrate allogeneic and syngeneic grafts were observed. First, mono-DCs were present in significantly higher numbers in allogeneic grafts and they persisted throughout the duration of the experiment (up to 6 weeks after transplantation). In contrast, mono-DC infiltration of syngeneic grafts was transient, reaching undetectable levels between 2 and 3 weeks after transplantation. Second, mono-DCs harvested from allogeneic grafts expressed IL-12 and stimulated both T-cell proliferation and differentiation to IFN $\gamma$ producing cells, while those harvested from syngeneic grafts did not express IL-12 and failed to induce IFN $\gamma$ production, despite triggering T-cell proliferation. IFN $\gamma$ production by $\mathrm{T}$ cells stimulated with mono-DCs from allogeneic grafts was similar to that measured in $\mathrm{T}$ cells stimulated with splenic DCs + LPS, indicating that allogeneic non-self has adjuvant properties similar to PAMPs. The findings also imply that allogeneic non-self is necessary for full maturation of monocytes to DCs that drive the Th1 response, while inflammation alone (associated with the transplantation of a syngeneic graft) is not sufficient. This conclusion is consistent with that of an earlier study in which the effects of inflammatory mediators and PAMPs on DC maturation were compared in vitro (45). In additional experiments, we also established that in vivo exposure of monocytes to allogeneic non-self precipitates T-cell-mediated rejection of single minor antigen-mismatched heart grafts that 
are otherwise accepted by the host and, conversely, that depletion of mono-DCs blunts allograft rejection. Therefore, akin to the Janeway paradigm of innate microbial recognition, innate sensing of allogeneic non-self induces the maturation of APCs that then trigger a productive immune response. Events associated with the transplantation procedure (namely, inflammation or danger caused by ischemia-reperfusion injury), which are common to both syngeneic and allogeneic grafts, are not sufficient.

\section{Innate non-self recognition in xenotransplantation}

Although the mechanisms by which the innate immune system detects allogeneic non-self are not currently known, some insights may be gleaned from xenotransplantation. In their studies of human hematopoietic stem cell (HSC) engraftment in immunodeficient mouse strains, Danska and colleagues (46) observed that NOD-SCID mice accepted human HSCs much more readily than equally immunodeficient mice on the B6 background. Through elegant genetic mapping experiments using NOD and NOR congenic and subcongenic strains, they established that polymorphisms in the SIRPa gene modulate engraftment of human HSC in mice. SIRPa is a polymorphic Ig superfamily receptor expressed on macrophages, monocytes, DCs, and neurons. SIRPa binds to a ubiquitously expressed ligand, CD47. CD47-SIRPa interaction mediates inhibitory signals, which lead to decreased phagocytosis by macrophages, reduced TNFa production, and diminished DC maturation (47). It is therefore likely that enhanced binding of NOD-SIRPa on mouse macrophages to CD47 on human cells enables better engraftment of human HSC in this particular mouse strain. Conversely, 'incompatibility' between donor CD47 and recipient SIRPa when tissues are transplanted between disparate animal species is responsible for phagocytosis of xenogeneic cells by host macrophages (48). The same group showed that CD47 could play an important role in one form of tolerance to allogeneic transplants whereby donor cells are transfused to the recipient to induce immunological unresponsiveness (49). While the relevance of these findings to allorecognition by the innate immune system is not known, immunoglobulin superfamily-like receptors mediating activating or inhibitory signals could be a prototypic molecular mechanism of how allogeneic non-self is sensed by monocytes.

\section{Concluding remarks}

There is mounting evidence that the mammalian innate immune system responds to allogeneic non-self independent of T, B, and NK cells. This form of allorecognition may initiate or amplify the responses of recipient $\mathrm{T}$ cells to allografts by inducing the maturation of APCs. It could also provide phagocytic cells with the means to kill allogeneic targets without inflicting damage on self-tissues. Fifty years ago, Sir Macfarlane Burnet suggested that: 'a viviparously produced embryo in the wrong place might become a damaging parasite' (50). Is it possible then that innate allorecognition systems have evolved to survey the maternal decidua for incipient invasion by fetal cells? Further studies are necessary to test this hypothesis and possibly link natural allogeneic encounters (pregnancy) to artificial ones (organ transplantation).

\section{Acknowledgments}

This work was supported by American Heart Association grant 11SDG7230011 to MHO, NIH grant AI099465 to FGL; and the Frank \& Athena Sarris Chair in Transplantation Biology (FGL). The authors have no conflicts of interest to declare.

\section{References}

1. Iwasaki A, Medzhitov R. Regulation of adaptive immunity by the innate immune system. Science. 2010; 327:291-295. [PubMed: 20075244] 
2. Palm NW, Medzhitov R. Pattern recognition receptors and control of adaptive immunity. Immunol Rev. 2009; 227:221-233. [PubMed: 19120487]

3. Beutler BA. TLRs and innate immunity. Blood. 2009; 113:1399-1407. [PubMed: 18757776]

4. Klein, J. Biology of the Mouse Histocompatibility-2 Complex. Springer-Verlag; New York, Heidelberg, Berlin: 1975. Function and evolution of the H-2 complex; p. 521-536.

5. Sykes, M.; Wood, K.; Sachs, DH. Transplantation Immunology. In: Paul, WE., editor. Fundamental Immunology. 6th edn. Woleters Kluwer/Lippincott Williams \& Wilkins; Philadelphia: 2008. p. 1426-1488.

6. Bonney EA, Matzinger P. The maternal immune system's interaction with circulating fetal cells. J Immunol. 1997; 158:40-47. [PubMed: 8977173]

7. Pearse AM, Swift K. Allograft theory: transmission of devil facial-tumour disease. Nature. 2006; 439:549. [PubMed: 16452970]

8. Murgia C, Pritchard JK, Kim SY, Fassati A, Weiss RA. Clonal origin and evolution of a transmissible cancer. Cell. 2006; 126:477-487. [PubMed: 16901782]

9. Felix NJ, et al. Alloreactive T cells respond specifically to multiple distinct peptide-MHC complexes. Nat Immunol. 2007; 8:388-397. [PubMed: 17322886]

10. Janeway CA Jr. Approaching the asymptote? Evolution and revolution in immunology. Cold Spring Harb Symp Quant Biol. 1989; 54(Pt 1):1-13. [PubMed: 2700931]

11. Medzhitov R, PrestonHurlburt P, Janeway CA. A human homologue of the Drosophila Toll protein signals activation of adaptive immunity. Nature. 1997; 388:394-397. [PubMed: 9237759]

12. Poltorak A, et al. Defective LPS signaling in $\mathrm{C} 3 \mathrm{H} / \mathrm{HeJ}$ and $\mathrm{C} 57 \mathrm{BL} / 10 \mathrm{ScCr}$ mice: mutations in $\mathrm{Tl} 4$ gene. Science. 1998; 282:2085-2088. [PubMed: 9851930]

13. Broz P, Monack DM. Newly described pattern recognition receptors team up against intracellular pathogens. Nat Rev Immunol. 2013; 13:551-565. [PubMed: 23846113]

14. Dierselhuis M, Goulmy E. The relevance of minor histocompatibility antigens in solid organ transplantation. Curr Opin Organ Transplant. 2009; 14:419-425. [PubMed: 19444105]

15. Rao DA, Pober JS. Endothelial injury, alarmins, and allograft rejection. Crit Rev Immunol. 2008; 28:229-248. [PubMed: 19024347]

16. Rock KL, Lai JJ, Kono H. Innate and adaptive immune responses to cell death. Immunol Rev. 2011; 243:191-205. [PubMed: 21884177]

17. Zelenay S, Reis e Sousa C. Adaptive immunity after cell death. Trends Immunol. 2013; 34:329335. [PubMed: 23608152]

18. Tsung A, et al. HMGB1 release induced by liver ischemia involves Toll-like receptor 4 dependent reactive oxygen species production and calcium-mediated signaling. J Exp Med. 2007; 204:29132923. [PubMed: 17984303]

19. Martinon F, Petrilli V, Mayor A, Tardivel A, Tschopp J. Gout-associated uric acid crystals activate the NALP3 inflammasome. Nature. 2006; 440:237-241. [PubMed: 16407889]

20. Chen CJ, Kono H, Golenbock D, Reed G, Akira S, Rock KL. Identification of a key pathway required for the sterile inflammatory response triggered by dying cells. Nat Med. 2007; 13:851856. [PubMed: 17572686]

21. Iyer SS, et al. Necrotic cells trigger a sterile inflammatory response through the Nlrp3 inflammasome. Proc Natl Acad Sci USA. 2009; 106:20388-20393. [PubMed: 19918053]

22. Sims GP, Rowe DC, Rietdijk ST, Herbst R, Coyle AJ. HMGB1 and RAGE in inflammation and cancer. Annu Rev Immunol. 2010; 28:367-388. [PubMed: 20192808]

23. Huang Y, et al. Extracellular hmgb1 functions as an innate immune-mediator implicated in murine cardiac allograft acute rejection. Am J Transplant. 2007; 7:799-808. [PubMed: 17331117]

24. Shi Y, Evans JE, Rock KL. Molecular identification of a danger signal that alerts the immune system to dying cells. Nature. 2003; 425:516-521. [PubMed: 14520412]

25. Shi Y, Galusha SA, Rock KL. Cutting edge: elimination of an endogenous adjuvant reduces the activation of CD8 T lymphocytes to transplanted cells and in an autoimmune diabetes model. J Immunol. 2006; 176:3905-3908. [PubMed: 16547223]

26. Honda K, Littman DR. The microbiome in infectious disease and inflammation. Annu Rev Immunol. 2012; 30:759-795. [PubMed: 22224764] 
27. Goldstein DR, Tesar BM, Akira S, Lakkis FG. Critical role of the Toll-like receptor signal adaptor protein MyD88 in acute allograft rejection. J Clin Invest. 2003; 111:1571-1578. [PubMed: 12750407]

28. Thornley TB, et al. TLR agonists abrogate costimulation blockade-induced prolongation of skin allografts. J Immunol. 2006; 176:1561-1570. [PubMed: 16424185]

29. Walker WE, Nasr IW, Camirand G, Tesar BM, Booth CJ, Goldstein DR. Absence of innate MyD88 signaling promotes inducible allograft acceptance. J Immunol. 2006; 177:5307-5316. [PubMed: 17015716]

30. Wang T, et al. Infection with the intracellular bacterium, Listeria monocytogenes, overrides established tolerance in a mouse cardiac allograft model. Am J Transplant. 2010; 10:1524-1533. [PubMed: 20642679]

31. Chakraverty R, et al. An inflammatory checkpoint regulates recruitment of graft-versus-host reactive T cells to peripheral tissues. J Exp Med. 2006; 203:2021-2031. [PubMed: 16880259]

32. Tesar BM, Zhang J, Li Q, Goldstein DR. TH1 immune responses to fully MHC mismatched allografts are diminished in the absence of MyD88, a toll-like receptor signal adaptor protein. Am J Transplant. 2004; 4:1429-1439. [PubMed: 15307830]

33. McKay D, Shigeoka A, Rubinstein M, Surh C, Sprent J. Simultaneous deletion of MyD88 and Trif delays major histocompatibility and minor antigen mismatch allograft rejection. Eur J Immunol. 2006; 36:1994-2002. [PubMed: 16874736]

34. Hutton MJ, Westwell-Roper C, Soukhatcheva G, Plesner A, Dutz JP, Verchere CB. Islet allograft rejection is independent of toll-like receptor signaling in mice. Transplantation. 2009; 88:10751080. [PubMed: 19898202]

35. Oberbarnscheidt MH, et al. Type I interferons are not critical for skin allograft rejection or the generation of donor-specific CD8+ memory T cells. Am J Transplant. 2010; 10:162-167. [PubMed: 19951284]

36. $\mathrm{Li} \mathrm{H}$, et al. Graft-versus-host disease is independent of innate signaling pathways triggered by pathogens in host hematopoietic cells. J Immunol. 2011; 186:230-241. [PubMed: 21098219]

37. Bingaman AW, et al. Vigorous allograft rejection in the absence of danger. J Immunol. 2000; 164:3065-3071. [PubMed: 10706695]

38. Anderson C, Carroll J, Gallucci S, Ridge J, Cheever A, Matzinger P. Testing time-, ignorance-, and danger-based models of tolerance. J Immunol. 2001; 166:3663-3671. [PubMed: 11238605]

39. Anderson CC, Matzinger P. Immunity or tolerance: opposite outcomes of microchimerism from skin grafts. Nat Med. 2001; 7:80-87. [PubMed: 11135620]

40. Chan WF, Perez-Diez A, Razavy H, Anderson CC. The ability of natural tolerance to be applied to allogeneic tissue: determinants and limits. Biol Direct. 2007; 2:10. [PubMed: 17437644]

41. Zecher D, et al. NK cells delay allograft rejection in lymphopenic hosts by downregulating the homeostatic proliferation of CD8+ T cells. J Immunol. 2010; 184:6649-6657. [PubMed: 20483732]

42. Li HW, Sachs J, Pichardo C, Bronson R, Zhao G, Sykes M. Nonalloreactive T cells prevent donor lymphocyte infusion- induced graft-versus-host disease by controlling microbial stimuli. J Immunol. 2012; 189:5572-5581. [PubMed: 23136200]

43. Zecher D, van Rooijen N, Rothstein D, Shlomchik W, Lakkis F. An Innate Response to Allogeneic Nonself Mediated by Monocytes. J Immunol. 2009; 183:7810-7816. [PubMed: 19923456]

44. Liu W, Xiao X, Demirci G, Madsen J, Li XC. Innate NK cells and macrophages recognize and reject allogeneic nonself in vivo via different mechanisms. J Immunol. 2012; 188:2703-2711. [PubMed: 22327074]

45. Sporri R, Reis e Sousa C. Inflammatory mediators are insufficient for full dendritic cell activation and promote expansion of CD4+ T cell populations lacking helper function. Nat Immunol. 2005; 6:163-170. [PubMed: 15654341]

46. Takenaka K, et al. Polymorphism in Sirpa modulates engraftment of human hematopoietic stem cells. Nat Immunol. 2007; 8:1313-1323. [PubMed: 17982459]

47. Barclay AN, van den Berg TK. The interaction between signal regulatory protein alpha (SIRPa) and CD47: structure, function, and therapeutic target. Annu Rev Immunol. 2014; 32:25-50. 
48. Ide K, et al. Role for CD47-SIRPalpha signaling in xenograft rejection by macrophages. Proc Natl Acad Sci USA. 2007; 104:5062-5066. [PubMed: 17360380]

49. Wang H, Wu X, Wang Y, Oldenborg PA, Yang YG. CD47 is required for suppression of allograft rejection by donor-specific transfusion. J Immunol. 2010; 184:3401-3407. [PubMed: 20208011]

50. Burnet FM. "Self-recognition" in colonial marine forms and flowering plants in relation to the evolution of immunity. Nature. 1971; 232:230-235. [PubMed: 4937075] 